\title{
EMERGENCE OF OSCILLATIONS AND SPATIO-TEMPORAL COHERENCE STATES IN A CONTINUUM-MODEL OF EXCITATORY AND INHIBITORY NEURONS
}

\author{
Silvio P. Sabatini and Fabio Solari \\ Department of Biophysical and Electronic Engineering \\ University of Genoa - Via all'Opera Pia 11a - I-16145 Genova-ITALY \\ silvio@dibe.unige.it -www.pspc.dibe.unige.it \\ Luca Secchi \\ Department of Electrical and Electronic Engineering \\ University of Cagliari - Piazza d'armi - I-09123 Cagliari-ITALY \\ luigi@diee.unica.it
}

\begin{abstract}
It is well known for a long time that, on a large-scale macroscopic scale, cortical activation states can be modeled by considering the dynamics of two non-linearly coupled populations of excitatory and inhibitory neurons [11], spatially organized into stereotyped modules. Considering the huge number of cells found in layered cortical areas neuronal activation is usually defined over spatially continuous domains (neural fields) as continuous distribution of neurons and synapses where each point in space corresponds to a neural population [6]. Formally, each layer dynamics is described by integro-differential ordinary equations, where the integral kernel is a smooth function that replaces the synaptic interconnection matrix and continuous position variables replace the indices of discrete models. In this way, metric relations apply and it becomes possible to model the geometrical properties, such as spatial nearness and topology, that characterize the rich anatomy structure of cortical areas (e.g., maps, columns, dendritic and axonal arborizations, cell density, etc.) [7] [4]. By introducing a diffusive coupling into the equations that govern the punctual excitation-inhibition reactions, additional local interactions take place, providing a gradual spread of the variations of activity in space and simulates the continuity constraint of the neural field. In general, reaction-diffusion systems are natural candidates to model cortical phenomenological processes associated to sensory information processing, since their diffusion components allow for a weighted averaging of the input signal over a region of a certain extent (i.e., the receptive field), thus realizing a reduction in the complexity of the signal, while the non-linear reaction components provide a flexible medium through which all sorts of excitatory and inhibitory behaviour can be modeled.
\end{abstract}

In this paper, we aimed to model the joint spatio-temporal oscillatory dynamics observed in the visual cortices [1] [9], through the behaviour of a field oscillatory system. To this purpose, we introduced a 2nd-order delay partial differential equation characterized by a point non-linear interaction similar to the König and Schillen's basic oscillatory element [5] and by a recurrent linear coupling, modeled by an excitatory diffusive term and lateral inhibition:

$$
\begin{aligned}
\tau_{0} \frac{\partial}{\partial t} e(x, t)= & -\alpha_{e} e(x, t)+D \frac{\partial^{2}}{\partial x^{2}} e(x, t)-b[e(x-d, t)+e(x+d, t)] \\
& -w_{i e} F\left[i\left(x, t-\tau_{i e}\right)\right]+s(x, t) \\
\tau_{0} \frac{\partial}{\partial t} i(x, t)= & -\alpha_{i} i(x, t)+w_{e i} F\left[e\left(x, t-\tau_{e i}\right)\right]
\end{aligned}
$$

where $e(x, t)$ and $i(x, t)$ are the excitatory and inhibitory activity, respectively; $s(x, t)$ is the driving input; $\tau_{0}$ is the membrane time constant; $\tau_{i e}$ and $\tau_{e i}$ are coupling delays; $w_{i e}$ and $w_{e i}$ are coupling weights between populations; $\alpha_{e}$ and $\alpha_{i}$ are damping constants; $D$ is the diffusion coefficient; $b$ is the lateral inhibition strength; $d$ is the lateral inhibition distance; $F(\cdot)$ is a sigmoidal output function. A balance between the excitatory and inhibitory influences can be controlled by acting jointly on the field parameters $b$ and $D$. Lateral inhibitory processes are, indeed, primed by large values of $b$, but their effects are spread over the neural field by diffusive processes, controlled by $D$. The resulting system behaves as a network of diffusion-coupled oscillators.

Considering the spatial integration properties, as mediated by the diffusion processes, we investigated the cooperative response to multiple activation sites in relation to the formation of coherently 
A

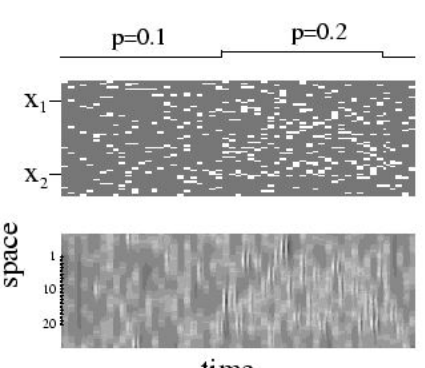

time

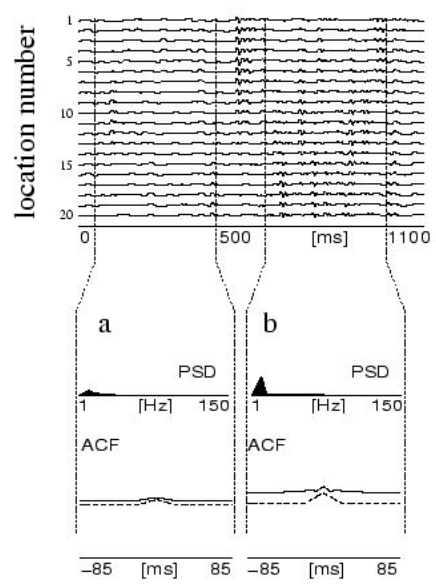

B
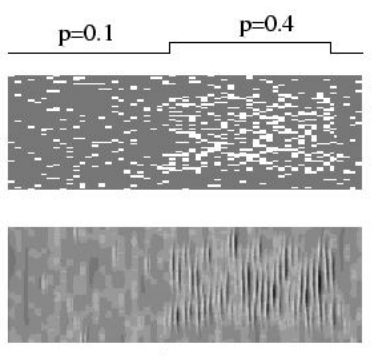

time

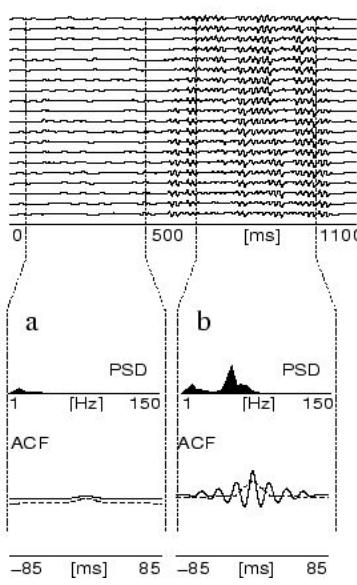

C
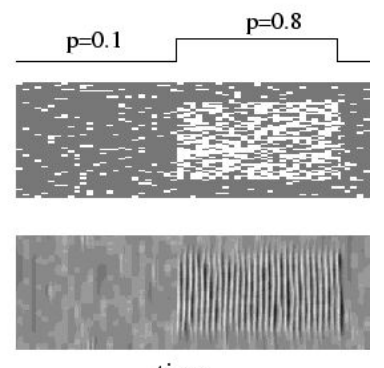

time

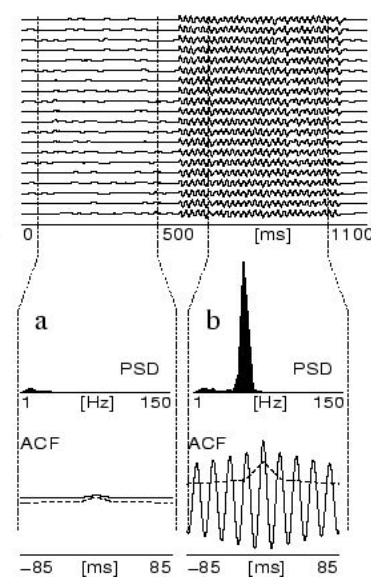

Figure 1: Stimulus-dependent responses obtained by stochastic input activation. Upper panels: grayscale spatio-temporal maps of the input configuration and of the corresponding cortical responses. Bright and dark grays represent excitatory and inhibitory activity, respectively. After 500 $\mathrm{ms}$, the onset of a specific stimulus, that lasts $500 \mathrm{~ms}$ more, increases the activation probability in the spatial domain $\left[x_{1}, x_{2}\right]$. Under condition of sufficient activation probability $p$ coherent spatiotemporal oscillations emerge over a large spatial region. Lower panels: LFP signal courses for the 20 nearby spatial locations evidenced in the leftmost spatio-temporal map response. Lowermost figures represent the corresponding average power spectra (PSDs) and the normalized autocorrelation functions (ACFs) for the 20 single response epochs delimited by dotted lines. a: $400 \mathrm{~ms}$ epochs before specific stimulation $(p=0.1)$, b: $400 \mathrm{~ms}$ epochs during specific stimulation $(p>0.1)$. Note the sharp peak in the PSD and the Gabor-like shape of the ACF obtained during specific stimulation. The dashed lines in the ACF graphs correspond to the average autocorrelations of the input signals.

oscillating assemblies. To this end, we consider, for multiple sites in space, volleys of afferent inputs, characterized by short periods (bursts) of continuous activity for sparse uniformly distributed sites of activation in space. Formally, for each spatial location in a given region, the input signal $s(x, t)$ is a stochastic process taking the value $A$ or 0 with probabilities $p$ and $(1-p)$, respectively, in each temporal interval. Augmenting the activation probability $p$ the temporal frequency of the volley increases, as well as the spatial density of the activation sites. Numerical simulations evidenced that rapid successive activation of multiple cortical sites ignites a cooperative reaction of the diffusion layer which results, for sufficiently high activation probability $p$, in the formation of coherent oscillatory states. Specifically, when the increment of the activation probability $p$ occurs in a spatially continuous cortical region, local groups of neurons engage in stimulus-specific responses as localized oscillatory assemblies (see Fig. 1).

Specific stimulation augments the activity of topologically continuous cortical locations and, under condition of sufficient excitatory drive, local oscillatory assemblies emerge. The resulting oscillations observed in the excitatory signal $e(x, t)$ closely resemble the time courses of real LFPs recorded in visual cortex [1] [9].

The presence of a diffusive medium operates so that the oscillatory activity elicited, through specific stimulations, at different locations of the cortical layer, results intrinsically coupled by diffusion, yielding large-scale areas of coherent activity. Diffusion and lateral inhibition play a key role in the synchronization and de-synchronization processes of the oscillatory activity, regulating 
the spatio-temporal properties of the resulting coherence fields. The spatial extension of the coherence fields depends on the intensity of lateral inhibition and on the degree of diffusion of the neural field. In particular, an increase of lateral inhibition disrupts spatio-temporal coherence, thus providing an intrinsic mechanism for de-synchronizing cortical activity. Low values of diffusion facilitate the formation of highly incoherent states, whereas high values of diffusion oppose desynchronization, thus providing an intrinsic synchronization mechanism. The projection to visual space of the coherence fields of the oscillatory assemblies may determine association fields [2] or context fields [8], in relation to their possible role in feature linking [10] and stimulus selection [3].

In conclusion, the major novelty of our model is the inclusion of the continuous space dimensionality that allows the system to exhibit cooperative activation properties both in time and space, by reacting to spatio-temporal input patterns with ordered oscillatory states in space and time. Specifically, we observed: (1) emergent local oscillatory assemblies, (2) intrinsic synchronization/desynchronization properties, (3) ordered spatial distributions of coherent states, that act as a flexible medium to establish feature association. A detailed analysis of the coherent rhythmic oscillations obtained through simulations evidenced the capability of the model of reproducing, with high accuracy, the macroscopic behaviour observed in real cortical networks.

Keywords: Neural fields, Cortical neurodynamics, Diffusively coupled oscillators.

\section{References}

[1] R. Eckhorn. Oscillatory and non-oscillatory synchronizations in the visual cortex and their possible roles in associations of visual features. In H.B.M. Uylings J. van Pelt, M.A. Coner and F.H. Lopes da Silva, editors, Progress in Brain Research, Vol 102, pages 405-426. Elsevier Science, 1994.

[2] R. Eckhorn, H.J. Reitböck, M. Arndt, and P. Dicke. Feature linking via synchronization among distribuited assemblies: Simulations of results from cat visual cortex. Neural Comp., 2:293-307, 1990.

[3] P. Fries, J-H. Schroeder, P.R. Roefsema, W. Singer, and A.K Engel. Oscillatory neural synchronisation in primary visual cortex as a correlate of stimulus selection. J. Neurosci, 22:3739-3754, 2002.

[4] Z.F. Kisvarday, D.S. Kim, U.T. Eysel, and T. Bonhoeffer. Relationship between lateral inhibitory connections and the topography of the orientation map in cat visual cortex. Eur. J. Neurosci., 6:1619-1632, 1994.

[5] P. König and T.B. Schillen. Stimulus-dependent assembly formation of oscillatory responses: I. synchronization. Neural. Comp., 3:155-166, 1991.

[6] H.A. Mallot and F. Giannakopoulos. Population networks: a large-scale framework for modelling cortical neural networks. Biol. Cybern., 75:441-452, 1996.

[7] H.A. Mallot, W. von Seelen, and F. Giannakopoulos. Neural mapping and space variant image processing. Neural Networks, 3:245-263, 1990.

[8] W.A. Phillips and W. Singer. In search of common foundations for cortical computation. Behav. Brain Sci., 20:657-722, 1997.

[9] W. Singer and C.M. Gray. Visual feature integration and the temporal correlation hypothesis. Ann. Rev. Neurosci., 18:555-586, 1995.

[10] D-L. Wang and D Terman. Locally excitatory globally inhibitory oscillator network. IEEE Trans. Neural Net., 6:283-286, 1995.

[11] H.R. Wilson and J.D. Cowan. Excitatory and inhibitory interactions in localized populations of model neurons. Biophysical Journal, 12:1-23, 1972. 